ARTICLE

Received 18 Oct 2016 | Accepted 14 Mar 2017 | Published 19 May 2017

DOI: $10.1038 /$ ncomms15269

OPEN

\title{
A high quantum yield molecule-protein complex fluorophore for near-infrared II imaging
}

\author{
Alexander L. Antaris ${ }^{1, \star}$, Hao Chen ${ }^{2,3, \star}$, Shuo Diao ${ }^{1}$, Zhuoran Ma1 ${ }^{1}$ Zhe Zhang ${ }^{3}$, Shoujun Zhu1, Joy Wang ${ }^{1}$, \\ Alexander X. Lozano ${ }^{1}$, Quli Fan ${ }^{3}$, Leila Chew ${ }^{1}$, Mark Zhu ${ }^{3}$, Kai Cheng ${ }^{3}$, Xuechuan Hong ${ }^{2}$, Hongjie Dai ${ }^{1}$ \\ \& Zhen Cheng $^{3}$
}

Fluorescence imaging in the second near-infrared window (NIR-II) allows visualization of deep anatomical features with an unprecedented degree of clarity. NIR-II fluorophores draw from a broad spectrum of materials spanning semiconducting nanomaterials to organic molecular dyes, yet unfortunately all water-soluble organic molecules with $>1,000 \mathrm{~nm}$ emission suffer from low quantum yields that have limited temporal resolution and penetration depth. Here, we report tailoring the supramolecular assemblies of protein complexes with a sulfonated NIR-II organic dye (CH-4T) to produce a brilliant 110-fold increase in fluorescence, resulting in the highest quantum yield molecular fluorophore thus far. The bright molecular complex allowed for the fastest video-rate imaging in the second NIR window with $\sim 50$-fold reduced exposure times at a fast 50 frames-per-second (FPS) capable of resolving mouse cardiac cycles. In addition, we demonstrate that the NIR-II molecular complexes are superior to clinically approved ICG for lymph node imaging deep within the mouse body.

\footnotetext{
${ }^{1}$ Department of Chemistry, Stanford University, Stanford, California 94305, USA. ${ }^{2}$ State Key Laboratory of Virology, Key Laboratory of Combinatorial Biosynthesis and Drug Discovery (Wuhan University), Ministry of Education, Wuhan University School of Pharmaceutical Sciences, Wuhan 430071, China. ${ }_{3}^{3}$ Molecular Imaging Program at Stanford (MIPS), Bio-X Program, and Department of Radiology, Canary Center at Stanford for Cancer Early Detection, Stanford University, Stanford, California 94305-5344, USA. * These authors contributed equally to this work. Correspondence and requests for materials should be addressed to Z.C. (email: zcheng@stanford.edu) or to H.D. (email: hdai@stanford.edu) or to X.H. (email: xhy78@whu.edu.cn).
} 
W ithin the last 5 years, the development of new fluorophores emitting in the second near-infrared widow (NIR-II) at wavelengths ranging from 1,000 to $1,700 \mathrm{~nm}$ has allowed visualization of deep anatomical features with an unprecedented degree of clarity ${ }^{1-11}$. Light scattering and attenuation as well as background autofluorescence all decrease when imaging at progressively longer wavelengths, which have enabled non-invasive through-skull imaging of brain vasculature, detecting tumours at depths of $\sim 4 \mathrm{~mm}$ in the brain, resolving blood flow dynamics resulting from cardiac waveforms, and assessing blood flow anomalies in cardiovascular disease and traumatic brain injury ${ }^{1-3,10}$. However, the majority of NIR-II fluorophores suffer from low quantum yields as the generation of long-wavelength photons require low bandgap materials in which non-radiative decay processes tend to dominate over radiative photon emission ${ }^{12}$. In the visible or NIR-I $(\sim 750-1,000 \mathrm{~nm})$ regions quantum yields of $\sim 80 \%$ and $\sim 10 \%$, respectively, are common yet those in the NIR-II are typically in the range of $\sim 0.01-1.4 \%$ with the exception of lead sulfide quantum dots ${ }^{1,13-16}$. The dearth of high quantum yield NIR-II fluorophores has hindered fully reaching the potential of deepimaging penetration depths in this window and caused poor temporal resolution as long exposure times are needed to compensate for low brightness. A clinically translatable high quantum yield organic NIR-II fluorophore would open up many exciting possibilities for biomedical fluorescence imaging.

While most current NIR-II fluorophores consist of inorganic semiconducting nanomaterials, the development of long- wavelength organic dyes similar in functionality and biocompatibility to those in the visible and NIR-I should allow NIR-II imaging to transition rapidly into a clinical setting and the broader research community. Donor-acceptor-donor (D-A-D) dyes are a promising class of small-molecule fluorophores for bioimaging whose emission extends past $1,000 \mathrm{~nm}^{11,17}$. Traditionally used for semiconducting organic electronics such as OLEDs and dye sensitized solar cells, this highly tunable dye architecture is built around a strong electron acceptor such as benzo[1,2-c:4,5-c' $]$ bis $([1,2,5]$ thiadiazole) (BBTD) to which strong electron donors are wired through $\pi$-spacers to lower the energy gap. Molecular engineering of the energy difference between the highest occupied molecular orbital (HOMO) and lowest unoccupied molecular orbital (LUMO) levels allows wavelength tuning of emission peaks spanning the NIR-II. However, the hydrophobic nature of these dyes requires additional functionalization to increase aqueous solubility. We recently reported the first organic dye for NIR-II imaging, a carboxylated D-A-D dye termed CH1055, which required PEGylation if not conjugated to a hydrophilic protein ${ }^{2}$. Although CH1055-PEG demonstrated excellent pharmacokinetics, a low quantum yield of $\sim 0.3 \%$, while typical of NIR-II fluorophores, leaves ample room for further improvement.

Herein, we demonstrate that changing the functional groups from carboxylic to sulfonic acid results in a completely watersoluble organic NIR-II dye (CH-4T) that readily forms supramolecular assemblies with plasma proteins to produce a brilliant increase in fluorescent brightness ${ }^{18}$. The fluorescence intensity of a

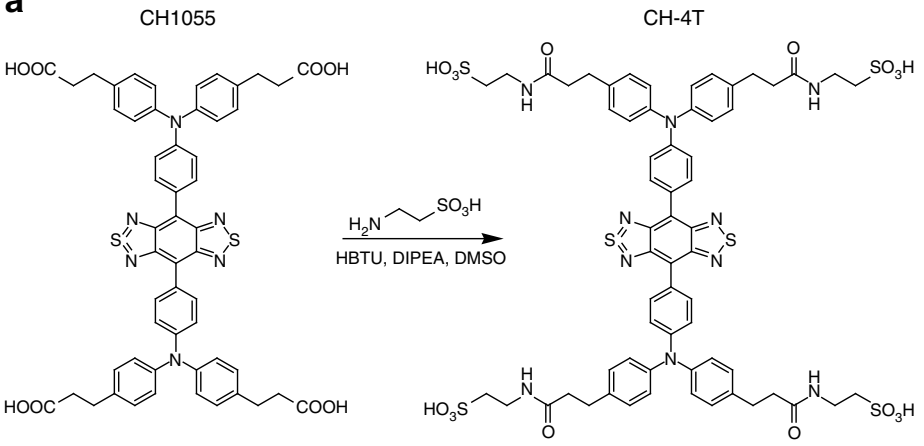

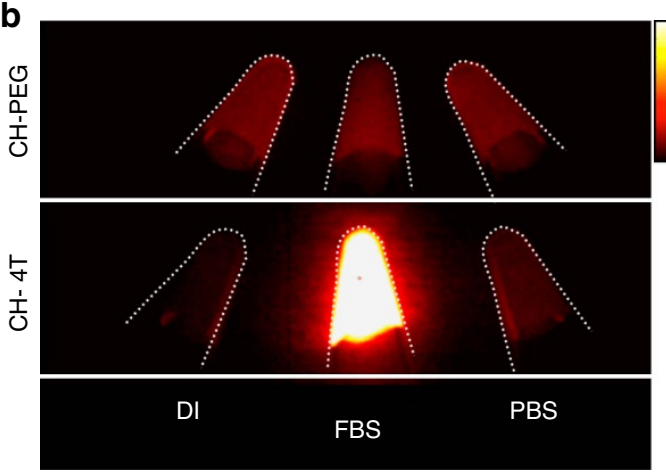

e

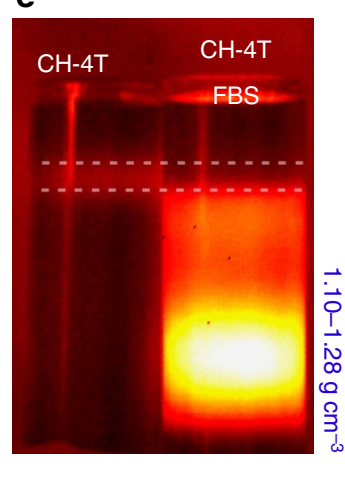

Figure 1 | Synthesis and optical characterization of CH-4T. (a) Chemical structure of $\mathrm{CH} 1055$ and the one-step sulfonation to produce $\mathrm{CH}-4 \mathrm{~T}$. (b) NIR-II fluorescent images (10 ms, 110OLP) of CH-PEG and CH-4T in deionized water, FBS and PBS at equivalent absorbance of OD 0.1 at $808 \mathrm{~nm}$. Colourbar next to $\mathbf{b}$ ranges from 1,000 to 15,000. (c) Fluorescent emission spectra of $\mathrm{CH}-\mathrm{PEG}$ and $\mathrm{CH}-4 \mathrm{~T}$ in FBS, PBS after excitation at $808 \mathrm{~nm}$. (d) Normalized fluorescent emission spectrum of CH-PEG in PBS and $\mathrm{CH}-4 \mathrm{~T}$ in FBS, PBS demonstrating $\sim 50 \mathrm{~nm}$ hypsochromic shift. (e) NIR-II fluorescent image (1000LP, $10 \mathrm{~ms}$ ) of centrifuge tubes after sucrose DGU with free $\mathrm{CH}-4 \mathrm{~T}$ dye on the left and $\mathrm{CH}-4 \mathrm{~T}$ pre-mixed with FBS on the right. Dotted white line indicates buoyant density region of unbound $\mathrm{CH}-4 \mathrm{~T}$ as seen in free dye band in left tube. Bright regions in right centrifuge tube correspond to $\mathrm{CH}-4 \mathrm{~T}$-protein complexes that are found at the higher buoyant densities typical of proteins $\left(\sim 1.10-1.28 \mathrm{~g} \mathrm{~cm}^{-3}\right)$. 
$\mathrm{CH}-4 \mathrm{~T}$ increases $\sim 50$-fold in serum compared to phosphatebuffered solution (PBS) and revealed an impressively high quantum yield of up to $\sim 5 \%$. Further, simply heating the dye to $70^{\circ} \mathrm{C}$ for $10 \mathrm{~min}$ in serum boosted its quantum yield up to $11 \%$, affording the highest reported quantum yield to date for a clinically suitable NIR-II fluorophore. Optimizing the brightness of $\mathrm{CH}-4 \mathrm{~T}$ through protein complexation prior to injection allowed for the fastest video-rate imaging in the NIR-II with $\sim 1.5-2 \mathrm{~ms}$ exposure times and ultra-fast 50 frames-per-second (FPS) dynamic imaging capable of resolving cardiac cycles with unprecedented temporal resolution. In addition, we demonstrate that lymph nodes deep within the mouse $(\sim 5-8 \mathrm{~mm})$ are clearly imaged in the NIR-II using the $\mathrm{CH}-4 \mathrm{~T}$ complex with imaging performance superior to indocyanine green (ICG).

\section{Results}

Synthesis and optical characterization. Synthesis of the sulfonated small-molecule organic dye $(\mathrm{CH}-4 \mathrm{~T}, 1.4 \mathrm{kDa}$, Fig. 1a) was achieved through amide bond formation between the carboxylated $\mathrm{CH} 1055$ and taurine by $O$-(benzotriazol-1-yl)- $N, N, N^{\prime}, N^{\prime}$ tetramethyluronium hexafluorophosphate (HBTU), N,N-diisopropylethlamine (DIPEA) coupling in DMSO (see Supplementary Methods for complete synthesis details). Taurine is a common biomolecule found throughout the body containing both an amine and sulfonate group, thus allowing facile modification of $\mathrm{CH} 1055$. After conjugation, $\mathrm{CH}-4 \mathrm{~T}$ was purified with HPLC and the eluted fractions analysed by matrix-assisted laser desorption ionizing time-of-flight mass spectrometry (MALDI-TOF-MS) to collect $\mathrm{CH} 1055$ conjugated to four taurine moieties (Supplementary Fig. 1). An nuclear magnetic resonance (NMR) spectrum further confirmed the synthesis and purity of $\mathrm{CH}-4 \mathrm{~T}$ (Supplementary Fig. 2). An ultraviolet-visible absorbance spectrum revealed a strong peak at $\sim 738 \mathrm{~nm}$ (Supplementary Fig. 3). The relative fluorescent brightness of $\mathrm{CH}-4 \mathrm{~T}$ was investigated by matching the absorbance at $808 \mathrm{~nm}$ (optical density (OD) 0.1) in deionized water (DI), PBS and fetal bovine serum (FBS) and imaging the vials on an indium-gallium-arsenide (InGaAs) camera under $808 \mathrm{~nm}$ excitation. The fluorescent brightness of $\mathrm{CH}-4 \mathrm{~T}$ in DI water was marginally higher than in PBS, yet a drastic 35-fold increase in fluorescence in the range of 900-1,500 nm was observed after mixing free $\mathrm{CH}-4 \mathrm{~T}$ dye with FBS (10 ms, 1100LP; Figs $1 \mathrm{~b}$ and 2e for higher image dynamic range; see Methods for details on $\mathrm{CH}-4 \mathrm{~T}$ spectroscopy). In contrast, CH1055-PEG mixed at equivalent concentrations in identical biological media produced a uniform NIR-II brightness across all solutions (Fig. 1b). A fluorescent emission spectrum collected for CH-4T and CH-PEG in both PBS and FBS under an excitation of $808 \mathrm{~nm}$ (using a NIR-II spectrophotometer that compensated for the variability in the detector quantum efficiency at different wavelengths (Fig. 1c)) demonstrated a higher fluorescence brightness of $\mathrm{CH}-4 \mathrm{~T}$ in serum than $\mathrm{CH}-\mathrm{PEG}$ by 16 -fold, again suggesting the $\mathrm{CH}-4 \mathrm{~T}$ dye became brilliantly bright in the presence of serum proteins. $\mathrm{CH}-4 \mathrm{~T}$ mixed with FBS from various sources all produced virtually the same level of fluorescence enhancement regardless of manufacturer (Supplementary Fig. 4).

To investigate $\mathrm{CH}-4 \mathrm{~T}$ binding to biomacromolecules, the normalized fluorescence emission spectrum of CH-PEG in PBS was compared to $\mathrm{CH}-4 \mathrm{~T}$ in both PBS and FBS. The fluorescence emission peak of $\mathrm{CH}-\mathrm{PEG}$ and $\mathrm{CH}-4 \mathrm{~T}$ in $\mathrm{PBS}$ occurred at $\sim 1,055 \mathrm{~nm}$ with emission from 900 to $\sim 1,400 \mathrm{~nm}$ as expected from previous $\mathrm{CH} 1055$ spectroscopic measurements. However, $\mathrm{CH}-4 \mathrm{~T}$ in serum yielded a hypsochromic shift of $\sim 50 \mathrm{~nm}$ which is indicative of non-covalent binding between the dye and proteins through hydrophobic van der Waals's interactions as well as ion pairing between the sulfonate groups and cationic amino acid residues such as histidine, lysine and arginine ${ }^{19}$.

To further verify the formation of $\mathrm{CH}-4 \mathrm{~T}$ complexes with plasma proteins, the free dye and $\mathrm{CH}-4 \mathrm{~T}$ pre-mixed with FBS (CH-4T/FBS) were subjected to isopycnic density gradient ultracentrifugation (DGU) (Fig. 1e; see Methods for complete DGU details). A linearly changing sucrose density gradient $\left(1.10-1.28 \mathrm{~g} \mathrm{~cm}^{-3}\right)$ was established along the length of the centrifuge tube that corresponds to the average buoyant density range of proteins. During ultracentrifugation, macromolecules migrate to a position in the gradient where their buoyant density matches that of the surrounding gradient. Free $\mathrm{CH}-4 \mathrm{~T}$ dyes formed a distinct band near $\sim 1.10 \mathrm{~g} \mathrm{~cm}^{-3}$, while for $\mathrm{CH}-4 \mathrm{~T} / \mathrm{FBS}$ a heterogeneous distribution of protein-bound fluorescenceenhanced $\mathrm{CH}-4 \mathrm{~T}$ was observed throughout the density gradient with a bright region at the bottom of the tube $\left(\sim 1.20 \mathrm{~g} \mathrm{~cm}^{-3}\right)$. The absence of fluorescence in the buoyant density range corresponding to free $\mathrm{CH}-4 \mathrm{~T}$ in the centrifuge tube containing $\mathrm{CH}-4 \mathrm{~T} / \mathrm{FBS}$ indicates near-zero free dye and a strong protein binding affinity ${ }^{20}$.

Optimizing the brightness of $\mathrm{CH}-4 \mathrm{~T}$-protein complexes. To develop an ultra-bright NIR-II probe by exploiting $\mathrm{CH}-4 \mathrm{~T}-$ protein interactions, common serum proteins were added in excess to $\mathrm{CH}-4 \mathrm{~T}$ to observe the increase in fluorescence enhancement under the NIR-II camera. Both human and bovine serum albumin (HSA and BSA) demonstrated strong fluorescence enhancement by 17 -fold yet the intensity was $\sim 2 \times$ lower than the dye in serum (Fig. 2a). Even weaker $\mathrm{CH}-4 \mathrm{~T}$ fluorescence occurred when mixed with IgG antibodies. A fluorescence titration was performed by increasing the HSA concentration while keeping $\mathrm{CH}-4 \mathrm{~T}$ at a constant $1 \mu \mathrm{M}$ to determine the approximate binding stoichiometry. As seen in Fig. 2b, the maximum fluorescence intensity occurred at approximately a 2:1 HSA:CH-4T molar ratio.

As the brightness of $\mathrm{CH}-4 \mathrm{~T}$ seems highly dependent on optimal binding between the dye and protein surfaces, heating of the complexes was attempted with the hopes of exposing $\mathrm{CH}-4 \mathrm{~T}$ to typically inaccessible hydrophobic domains located in the protein interior. After heating for $10 \mathrm{~min}$, the brightness of the vials containing the $\mathrm{CH}-4 \mathrm{~T}$ and FBS mix increased steadily with temperature until $\sim 75-85^{\circ} \mathrm{C}$. However, heating to temperatures above $\sim 85^{\circ} \mathrm{C}$ caused a precipitous drop in fluorescent intensity. Significant aggregation was observed for temperatures above $\sim 90^{\circ} \mathrm{C}$, while a minimal amount of aggregation at lower temperatures was removed through centrifugation post-heating (30 min, 15,000 r.p.m.). Heating virtually every dye-protein complex caused a marked increase in fluorescent intensity with a similar $\sim 50 \mathrm{~nm}$ hypsochromic shift from $\sim 1,050$ to $\sim 1,000 \mathrm{~nm}$. After returning to room temperature, the thermally stabilized dye-protein complexes maintained their boosted brightness when compared to solely mixed solutions.

The CH-4T-protein complexes represented the brightest fluorescent agent based on organic molecules with an emission peak in the $\sim 1,000-1,100$ region of the NIR-II. Comparing the brightness of fluorophores in vials and cuvettes with the NIR-II camera may cause errors due to inter-filter effects, while the hypsochromic emission peak shift coupled with the nonlinear camera quantum efficiency as a function of wavelength necessitates a more rigorous measurement. The relative fluorescence brightness of $\mathrm{CH}-4 \mathrm{~T} / \mathrm{FBS}$ heated to $70^{\circ} \mathrm{C}$ for $10 \mathrm{~min}$, termed CH-4T/FBS-HT, was 28-fold, 36-fold and 22-fold brighter than carbon nanotubes (QY $=0.4 \%$; IR-26 $=0.5 \%)$, CH-PEG $(\mathrm{QY}=0.3 \%$; IR-26=0.5\%) and IR-26 $(\mathrm{QY}=0.5 \%)$ in $\mathrm{DCE}$, 
a

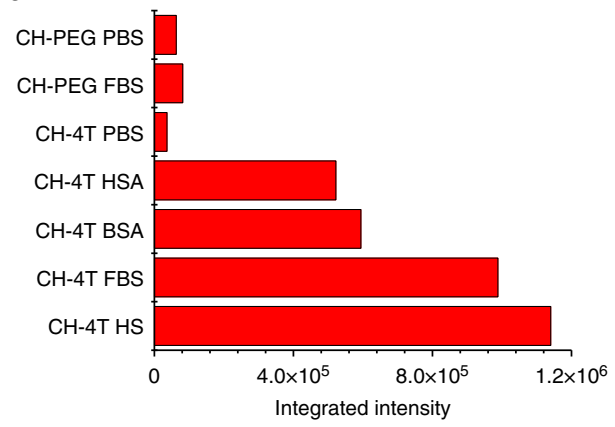

b

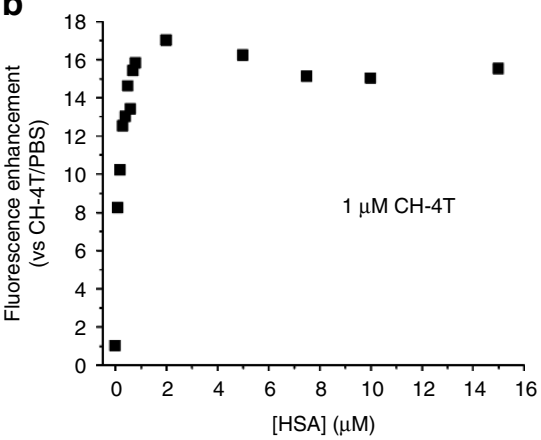

C

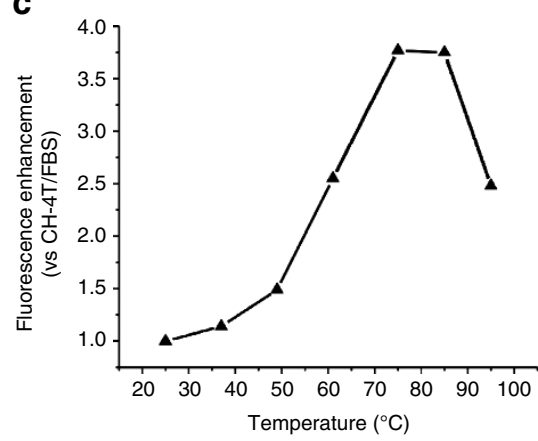

d

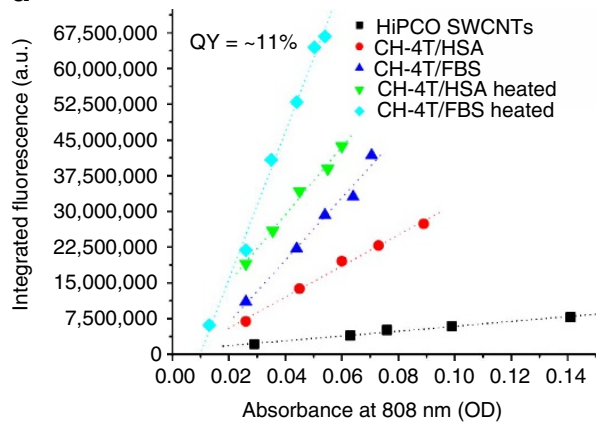

e
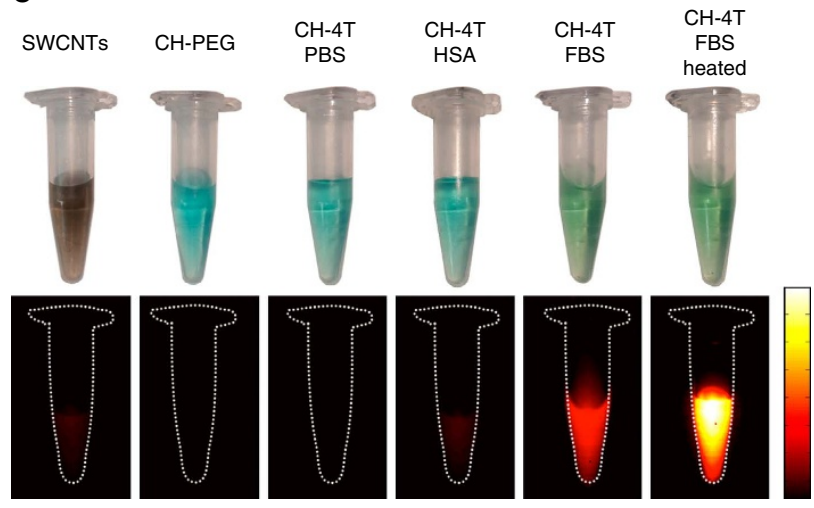

Figure 2 | Ultra-high quantum yield CH-4T-protein complexes. (a) Fluorescent brightness of $\mathrm{CH}-\mathrm{PEG}, \mathrm{CH}-4 \mathrm{~T}$ in protein solutions at 5:1 protein-to-dye molar ratio (b) Fluorescent titration for constant $1 \mu \mathrm{M}$ of $\mathrm{CH}-4 \mathrm{~T}$ in increasing HSA receptor concentration. (c) Plot demonstrating fluorescence brightening through heating for 10 min of $\mathrm{CH}-4 \mathrm{~T}$ pre-mixed with FBS and horn sonicated FBS. (d) Plot of the integrated fluorescence spectrum of $\mathrm{CH}-4 \mathrm{~T}$ pre-mixed with $\mathrm{HSA}$, FBS before and after heating at $70^{\circ} \mathrm{C}$ for $10 \mathrm{~min}$ at five different concentrations. Linear fits were used to calculate quantum yield by comparing the slopes to reference HiPCO SWCNTs (QY $=0.4 \%)(\mathbf{e})$ Photographs and corresponding NIR-Il fluorescent images (10 ms, $1000 \mathrm{LP})$ of carbon nanotubes, $\mathrm{CH}-\mathrm{PEG}$ and $\mathrm{CH}-4 \mathrm{~T}$ in HSA, FBS and heated FBS. Photographs were taken at an absorbance value of OD 1 at $808 \mathrm{~nm}$, while NIR-II fluorescent images were at the lower concentration of OD 0.1. Colourbar next to e ranges from 4,000 to 40,000.

respectively, with a 110 -fold brightness difference between $\mathrm{CH}-4 \mathrm{~T} / \mathrm{PBS}$ and $\mathrm{CH}-4 \mathrm{~T} / \mathrm{FBS}-\mathrm{HT}$ when measured on a wavelength-corrected NIR-II spectrophotometer (see Methods; Supplementary Fig. 5; Supplementary Tables 1 and 2 for enhancement calculations). Due to recent discrepancies in the reported quantum yield of the IR-26 reference fluorophore ( $\mathrm{QY}_{\text {IR26 }} \sim 0.05-0.5 \%$; see Discussion), the absolute quantum yields of $\mathrm{CH}-4 \mathrm{~T} / \mathrm{PBS}, \mathrm{CH}-4 \mathrm{~T} / \mathrm{FBS}$ and $\mathrm{CH}-4 \mathrm{~T} / \mathrm{FBS}-\mathrm{HT}$ are in the range of $0.0098-0.098 \%, 0.48-4.8 \%$ and $1.8-10.8 \%$, respectively. The NIR-II fluorescent images (1000LP, $10 \mathrm{~ms}$ ) in Fig. 2e qualitatively shows the disparate brightness levels between the brilliantly fluorescent $\mathrm{CH}-4 \mathrm{~T}$-protein complexes and current NIR-II contrast agents such as single-walled carbon nanotubes (SWCNTs) and CH-PEG, all with matching absorbance at $808 \mathrm{~nm}$ (OD 0.1).
Ultra-fast in vivo imaging with $\mathrm{CH}-4 \mathrm{~T}$. To investigate the in vivo optical properties of free $\mathrm{CH}-4 \mathrm{~T}$ dyes and the brightness optimized $\mathrm{CH}-4 \mathrm{~T}$-protein complexes, video-rate imaging (1000LP, $35 \mathrm{~ms}$ exposure time) of mouse hindlimb vasculature was performed after an intravenous injection of free $\mathrm{CH}-4 \mathrm{~T}$ and $\mathrm{CH}-4 \mathrm{~T} / \mathrm{FBS}-\mathrm{HT}$ at equivalent dosages $(90 \mu \mathrm{g}, 100 \mu \mathrm{l}$ at OD 3.2 at $808 \mathrm{~nm}$ ) under $808 \mathrm{~nm}$ excitation at a power density of $140 \mathrm{~mW} \mathrm{~cm}^{-2}$. After $10 \mathrm{~min}$ post-injection, a clear difference in the fluorescent intensity of the femoral vasculature was discerned as seen in Fig. 3a,b indicating that the higher quantum yield of pre-made complexes of $\mathrm{CH}-4 \mathrm{~T} / \mathrm{FBS}-\mathrm{HT}$ compared to $\mathrm{CH}-4 \mathrm{~T}$ that complexed with serum proteins in vivo in circulating blood upon injection of free $\mathrm{CH}-4 \mathrm{~T}$ generated a brighter contrast agent. The $\sim 2 \times$ brighter femoral artery of $\mathrm{CH}-4 \mathrm{~T} / \mathrm{FBS}-\mathrm{HT}$ injected mice compared to those injected with free dye correlates with the 
a

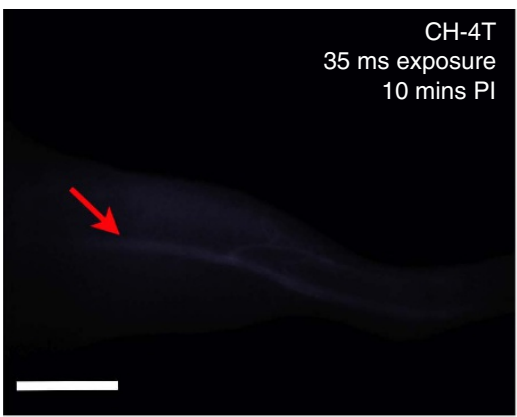

d

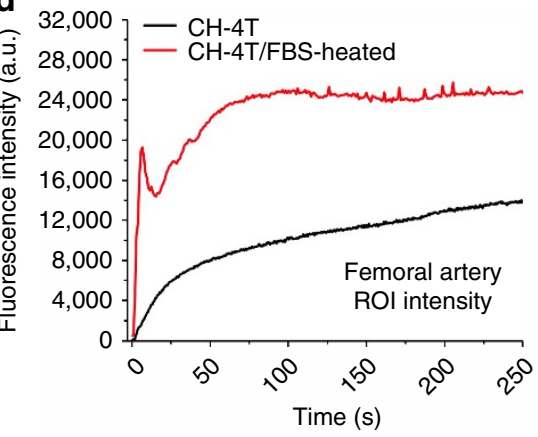

g

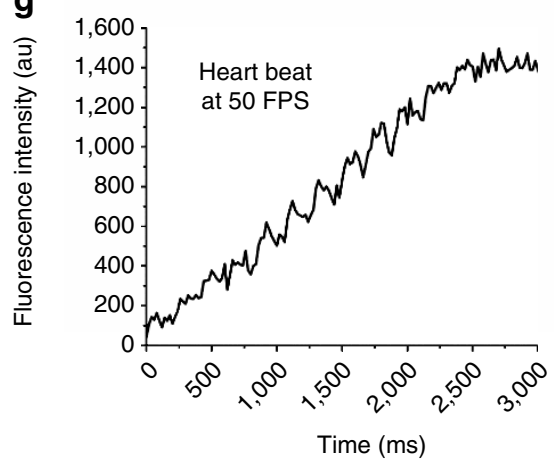

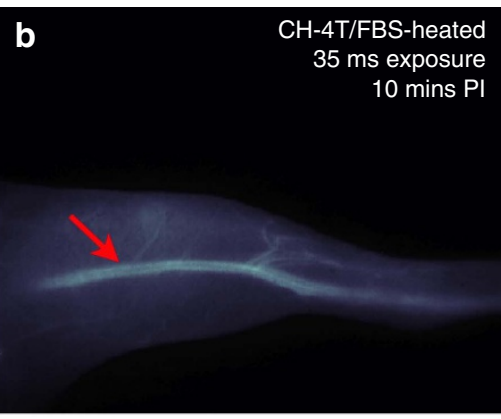
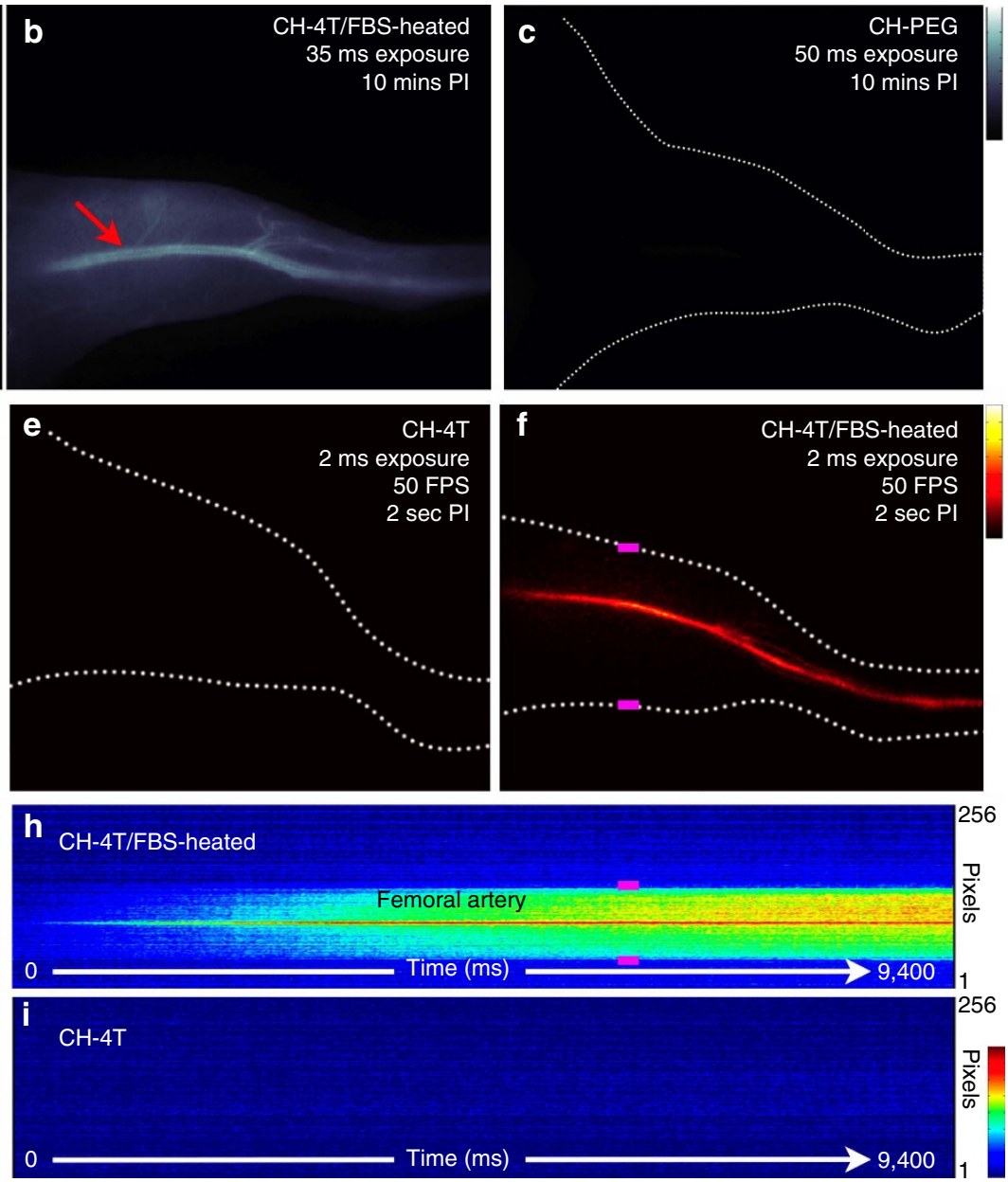

Figure 3 | Free CH-4T versus CH-4T-protein complexes for NIR-II hindlimb vasculature imaging. NIR-II fluorescent images of mouse hindlimb vasculature $10 \mathrm{~min}$ post-injection of (a) free $\mathrm{CH}-4 \mathrm{~T}$, (b) $\mathrm{CH}-4 \mathrm{~T}$ heated at $70^{\circ} \mathrm{C}$ for $10 \mathrm{~min}$ in $\mathrm{FBS}(\mathrm{CH}-4 \mathrm{~T} / \mathrm{FBS}-\mathrm{HT})$ and $(\mathbf{c}) \mathrm{CH}-\mathrm{PEG}$. All injection doses were normalized to an absorbance of OD 3.2 at $808 \mathrm{~nm}$ with an exposure time of $35 \mathrm{~ms}, 50 \mathrm{~ms}$ for $\mathrm{CH}-4 \mathrm{~T}, \mathrm{CH}-\mathrm{PEG}$, respectively. Scale bar in a of $0.5 \mathrm{~cm}$ and corresponds to $\mathbf{a}-\mathbf{c}, \mathbf{e}, \mathbf{f}$. Colourbar next to $\mathbf{c}$ ranges from 0.5 to $5 \times 10^{4}$ and corresponds to a-d. Fluorescent intensity of integrated ROI region on the hindlimb femoral artery plotted for $250 \mathrm{~s}$ post-injection during video-rate imaging of free $\mathrm{CH}-4 \mathrm{~T}$ and $\mathrm{CH}-4 \mathrm{~T} / \mathrm{FBS}-\mathrm{HT}$ ( $35 \mathrm{~ms}$ exposure time). NIR-II fluorescent images $2 \mathrm{~s}$ post-injection of $\mathbf{e}$ free $\mathrm{CH}-4 \mathrm{~T}$ and $\mathbf{f} \mathrm{CH}-4 \mathrm{~T} / \mathrm{FBS}-\mathrm{HT}$ during ultra-fast 50 FPS imaging. Colourbar next to $\mathbf{f}$ ranges from 500 to $-5,000$ and corresponds to e,f,g. Fluorescent intensity of integrated ROI region on the femoral artery for $3 \mathrm{~s}$ post-injection demonstrating a clearly resolvable cardiac cycle. The fluorescence intensity of a single vertical row of pixels during 50 FPS imaging in e,f was plotted against time for $\mathbf{h}$ CH-4T/FBS-HT and i free $\mathrm{CH}-4 \mathrm{~T}$ during $9.4 \mathrm{~s}$ post-injection. Pink bars in $\mathbf{f}, \mathbf{h}$ mark the edges of the hindlimb. The femoral artery is clearly visible as the solid red streak in $\mathbf{h}$ only after injection of pre-bound $\mathrm{CH}-4 \mathrm{~T}$ in heated FBS. Colourbar next to $\mathbf{i}$ ranges from 0 to 2,000 and corresponds to h,i. All NIR-II images and fluorescent intensity profiles for e-i were collected at a 2 ms exposure time. All NIR-II imaging was performed with a 1,000 nm long-pass emission filter.

$\sim 2-3 \times$ boosted solution brightness difference observed between $\mathrm{CH}-4 \mathrm{~T} / \mathrm{FBS}-\mathrm{HT}$ and the free dye mixed in serum. For comparison, much weaker fluorescence was observed at any time from a mouse injected with an equivalent CH-PEG dosage $(100 \mu \mathrm{l}$ at $\mathrm{OD} 3.2$ at $808 \mathrm{~nm}$; Fig. 3c), even at higher exposure times of $50 \mathrm{~ms}$, since $\mathrm{CH}-\mathrm{PEG}$ showed no complexing with serum proteins to afford enhanced fluorescence in vivo.

While the CH-4T/FBS-HT complex yielded a $\sim 2 \times$ increase in the steady-state vessel fluorescent intensity compared to free dye, the benefits of pre-optimizing the brightness of the $\mathrm{CH}-4 \mathrm{~T}-$ protein complexes are highly desired for imaging immediately post-injection. In Fig. 3d, the integrated intensity of a background subtracted region of interest (ROI) region in the femoral artery was plotted for the first $250 \mathrm{~s}$ post-injection for $\mathrm{CH}-4 \mathrm{~T}$ and $\mathrm{CH}-$ 4T/FBS-HT (position of ROI region indicated by red arrows in
Fig. 3a,b). During the first $10 \mathrm{~s}$ post-injection, a strikingly stronger rise in fluorescence was observed from the femoral artery of mice injected with the pre-brightened complex compared to free $\mathrm{CH}-4 \mathrm{~T}$. In contrast, the gradual increase in fluorescent intensity observed from the femoral artery of the mouse injected with free $\mathrm{CH}-4 \mathrm{~T}$ occurred as the brightening kinetics of the free dye required longer periods of time to binds to serum proteins during circulation to reach its full fluorescent potential brightness. By dividing the fluorescent intensity of the femoral artery of the pre-bound versus free dye (Supplementary Fig. 6), earlier time points demonstrate a $\sim 33$-fold higher brightness that equilibrates to the steady-state brightness difference of $\sim 2$-fold at $\sim 200 \mathrm{~s}$. After this point, the vessel intensities of mice injected with free $\mathrm{CH}-4 \mathrm{~T}$ have formed stable complexes and reached their steady-state maximum fluorescent brightness. The brightness 
difference between the femoral artery of $\mathrm{CH}-4 \mathrm{~T}$ and the prebrightened complex injected mice now matches the brightness difference seen at longer time points post-injection in Fig. 3a,b. In strong contrast to $\mathrm{CH}-4 \mathrm{~T}, \mathrm{CH}-\mathrm{PEG}$ at an equivalent dose produced a barely detectable increase in fluorescent signal in the femoral artery after injection that remained at a constant low fluorescent intensity (Supplementary Fig. 7).

The markedly higher brightness of pre-bound $\mathrm{CH}-4 \mathrm{~T}$ circulating in the vascular system during the first seconds post-injection can be exploited for high temporal resolution imaging of hemodynamic processes. A stark difference between injecting free $\mathrm{CH}-4 \mathrm{~T}$ and $\mathrm{CH}-4 \mathrm{~T} / \mathrm{FBS}-\mathrm{HT} \quad(250 \mu \mathrm{g} \mathrm{CH}-4 \mathrm{~T} / \mathrm{mouse})$ is clearly seen in the selected frames at equivalent time points during ultra-fast 50 FPS video imaging using exposure times of only 1.5-2 s (Fig. 3e,f) with an instrument overhead time of $\sim 19 \mathrm{~ms}$. At $2 \mathrm{~s}$ post-injection, no fluorescence is noted from the vasculature of mice injected with free $\mathrm{CH}-4 \mathrm{~T}$ due to the slow brightening kinetics of the dye in vivo. In contrast, the femoral artery is clearly resolved during 50 FPS imaging when injecting CH-4T/FBS-HT (Fig. 3e). Plotting the integrated ROI intensity of the femoral artery for $3 \mathrm{~s}$ post-injection of $\mathrm{CH}-4 \mathrm{~T} / \mathrm{FBS}-\mathrm{HT}$ allows for precise imaging of a cardiac cycle where intensity peaks corresponding to ventricular ejections (Fig. $3 g$ ) are resolved with much higher resolution compared to previous attempts with lower quantum yield semiconducting NIR-II polymers (see Supplementary Fig. 8 for comparison) ${ }^{1}$. The fluorescent intensity from a single row of pixels crossing the hindlimb was selected from each frame for the first $9.4 \mathrm{~s}$ of the 50 FPS videos that generated the images seen in Fig. $3 \mathrm{e}, \mathrm{f}$ and plotted as a function of time (Fig. 3h,i). The pink position markers denote the edges of the hindlimb in Fig. 3f,h. Plotting the fluorescent intensity line profile of a single row of pixels over time clearly shows that the blood flow front along with the femoral artery, visualized as the strong red streak in Fig. $3 \mathrm{~h}$, can easily be discerned with the $\mathrm{CH}-4 \mathrm{~T} / \mathrm{FBS}-\mathrm{HT}$ contrast agent yet virtually no fluorescence can be observed during the first $\sim 10 \mathrm{~s}$ when injecting unbound $\mathrm{CH}-4 \mathrm{~T}$ in Fig. 3i.

Deep lymph node imaging with $\mathrm{CH}-4 \mathrm{~T}$ complexes. To further investigate the advantages of high-brightness contrast agents for biomedical fluorescence imaging, the imaging quality of lymphatic vasculature and nodes were compared in both the first and second NIR windows. The popliteal and sacral lymph nodes were imaged in nude mice in a prone position with fluorophores injected in both footpads as seen in Fig. 4a. ICG dispersed in DI water at $50 \mu \mathrm{M}$ was utilized as a NIR-I contrast agent and lymph node-to-background ratios of $8.6 \pm 2.9$ were observed (Fig. 4b) which is consistent with reported values ${ }^{21}$. Although ICG can form complexes when mixed with proteins such as HSA, the benefits of the premixed ICG/HSA complex compared to free ICG in terms of lymph node detection efficiency is unclear ${ }^{21,22}$. Lymph node fluorescence from ICG and ICG/HSA-HT likewise produced nearly identical intensities as seen in Supplementary Fig. 9. However, a very notable difference in brightness of $\sim 8$-fold is observed between free $\mathrm{CH}-4 \mathrm{~T}$ injected on the left footpad and $\mathrm{CH}-4 \mathrm{~T} / \mathrm{HSA}-\mathrm{HT}$ injected on the right side footpad (Fig. 4c) at equivalent $50 \mu \mathrm{M}$ dosages immediately post-injection. The disparate fluorescent intensities of the popliteal and sacral lymph nodes for free $\mathrm{CH}-4 \mathrm{~T}$ and $\mathrm{CH}-4 \mathrm{~T} / \mathrm{HSA}-\mathrm{HT}$ demonstrated the need for premixing to form ultra-bright complexes for NIR-II lymphography. CH-4T/HSA-HT resulted in lymph node-tobackground ratios of $29 \pm 7.6$ with significantly sharper lymph node features with a popliteal/sacral FWHM of $2.52 \pm 0.23 \mathrm{~mm} /$ $1.61 \pm 0.11 \mathrm{~mm}$ compared to $4.86 \pm 0.17 \mathrm{~mm} / 4.4 \pm 0.29 \mathrm{~mm}$ with ICG in the NIR-I.
To demonstrate the enhanced clarity of features deep inside the body by imaging in the NIR-II, the lumbar lymph nodes were visualized after the belly was stretched to reduce the distance between the lumbar lymph nodes and the surface of the mouse (Fig. 4d). The lumbar lymph nodes sit on either side of the spine, laterally of the descending aorta bifurcation when observing the mouse in a supine position and are connected to a lymphatic channel responsible for draining the footpad and leg (see Supplementary Fig. 10 for lymphatic system details). Without contortion, the distance between the lumbar lymph nodes and the surface approaches $\sim 1-1.5 \mathrm{~cm}$, the bulk of which is mostly comprised of the intestines as shown in the illustration in Fig. 4e. Stretching the belly provides a fixed imaging depth of $\sim 0.5-0.8 \mathrm{~cm}$ and allows sufficient lymph node fluorescent signal in both windows (see Methods for details).

ICG $(50 \mu \mathrm{M})$ was injected into the footpad and digits on both sides; afterwards the mouse was fixed physically and stretched on the imaging platform until the lumbar lymph nodes were visualized with a silicon detector in the NIR-I (300 ms, $\quad 785-900 \mathrm{~nm}$; for imaging set-up details see Supplementary Figs 11 and 12). The subsequent injection of $\mathrm{CH}-4 \mathrm{~T} / \mathrm{HSA}-\mathrm{HT}(50 \mu \mathrm{M})$ on the same mouse in an identical manner and imaging on the InGaAs detector ( $400 \mathrm{~ms}, 1,100+\mathrm{nm}$ ) demonstrated a striking increase in the resolution of the lumbar lymph nodes as seen in Fig. 4f. Imaging with the $\mathrm{CH}-4 \mathrm{~T}$-protein contrast agent allowed the resolution of individual nodes with a signal-to-background (SBR) ratio of $\sim 13$ as opposed to the diffuse conjoined features observed in the NIR-I with an SBR of $\sim 2.5$. The enhanced clarity is reflected in the fluorescence cross-sectional intensity profile of the lumbar lymph nodes in both windows in Fig. $4 \mathrm{~g}$ as well as the $\sim 5 \times$ lower background autofluorescence levels in the NIR-II. Imaging in both windows was performed with a $785 \mathrm{~nm}$ laser $\left(16 \mathrm{~mW} \mathrm{~cm}^{-2}\right)$ to provide a constant excitation power density for both dyes.

\section{Discussion}

Imaging in the second near-infrared window allows optical imaging to peer deeper into the body with more clarity than any other wavelength regime ${ }^{22-27}$. However, the development of small-molecule organic dyes is necessary preceding the widespread use of this imaging technique. Inorganic nanomaterials are excellent for fluorescence-based preclinical animal imaging and while many current nanomaterial NIR-II probes show strong promise for clinical translation, organic NIR-II dyes similar in structure and pharmacokinetics to the FDA approved NIR-I organic fluorophores such as ICG and methylene blue should expedite the transition of NIR-II imaging into the clinic ${ }^{28-30}$. Researchers without the specialized knowledge of nanomaterial-based fluorophores would benefit from organic NIR-II dyes that could be incorporated into bioimaging procedures currently relying on the plethora of visible and NIR-I dyes. CH-4T's complete aqueous solubility combined with its high serum quantum yield makes it an attractive NIR-II clinical candidate capable of being utilized with ease for biomedical vascular imaging procedures. $\mathrm{CH}-4 \mathrm{~T}$ can dramatically reduce fluorescent dye doses by $\sim 25$-fold for biomedical imaging compared to previous, low quantum yield contrast agents such as CH-PEG. CH-4T can also uniquely enable the fastest temporal resolution in the NIR-II spectral window

The D-A-D NIR-II dye architecture shows strong potential for developing an array of NIR-II fluorescent probes through the molecular engineering of their constituent components. The addition of the negatively charged sulfonate groups translated into a dramatic boost in the in vivo quantum yield of $\mathrm{CH} 1055$ that allowed for video-rate imaging at the fastest frame rate along 
a

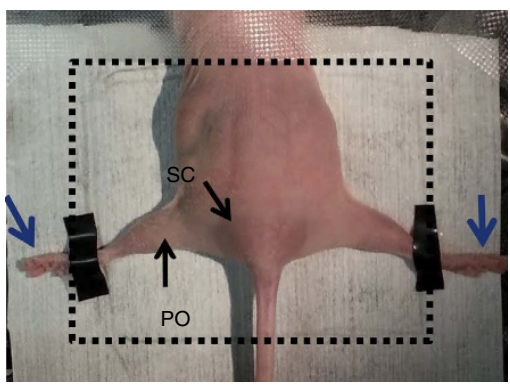

b

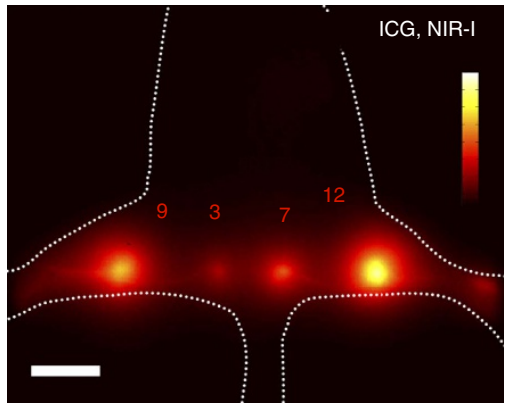

f

ICG, 785-900 nm

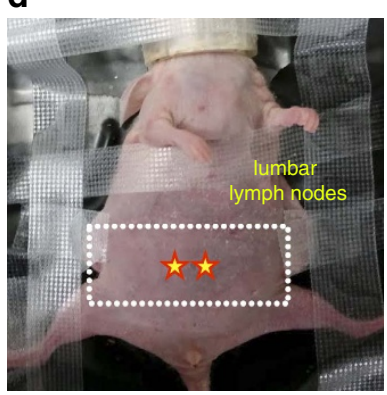

e

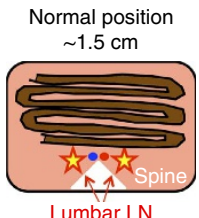

tretched position $\sim 0.5 \mathrm{~cm}$

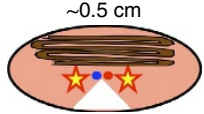

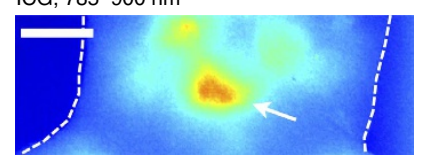

$\mathrm{CH}-4 \mathrm{~T} / \mathrm{HSA}$-heated, $1,100 \mathrm{~nm}$

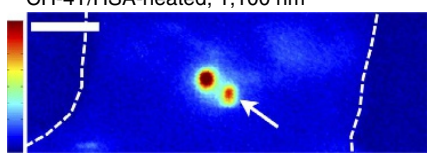

C

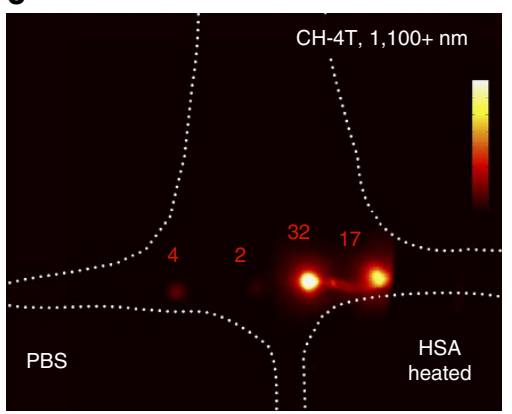

9

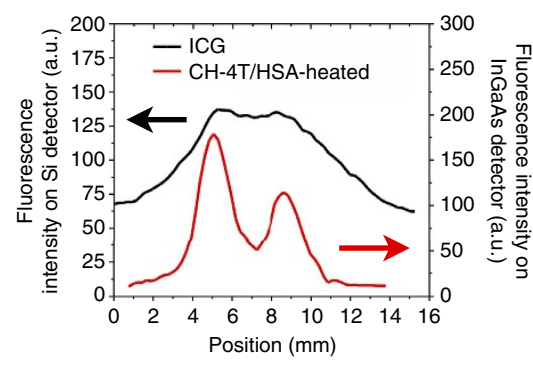

Figure 4 | Lymph node imaging with ultra-bright CH-4T-protein complexes. (a) Photograph depicting nude mouse in prone position for imaging popliteal and sacral lymph nodes in $\mathbf{b}, \mathbf{c}$. Fluorophore injection sites indicated by blue arrows next to footpad. (b) NIR-I fluorescent image $\sim 30$ min post-injection of $50 \mu \mathrm{M} \mathrm{ICG} \mathrm{(50} \mathrm{ms} \mathrm{exposure;} \mathrm{colourbar} \mathrm{ranges} \mathrm{from} \mathrm{4,000} \mathrm{to} \mathrm{40,000).} \mathrm{(c)} \mathrm{NIR-II} \mathrm{fluorescent} \mathrm{image} 30$ min post-injection of CH-4T/PBS injected in left foot and $\mathrm{CH}-4 \mathrm{~T} / \mathrm{HSA}-\mathrm{HT}$ in right foot at equivalent dosages of $50 \mu \mathrm{M} \mathrm{CH}-4 \mathrm{~T}$ (100 ms exposure; colourbar ranges from 4,000 to 55,000 ). $1 \mathrm{~cm}$ scale bar in b corresponds to both b,c. (d) Photograph depicting nude mouse in a stretched position to narrow the distance between lumbar lymph nodes and mouse surface. Stars indicate position of lumbar lymph nodes and white box corresponds to imaging field of view (e). Illustration of mouse anatomy surrounding lumbar lymph nodes demonstrating a change in imaging depth from $\sim 1.5$ to $\sim 0.5 \mathrm{~cm}$. White triangle corresponds to the spine with red and blue circles representing descending aorta and inferior vena cava, respectively. Brown lines represent intestines. (f) NIR-I/II fluorescent images of lumbar lymph nodes after $50 \mu \mathrm{M}$ of ICG and $\mathrm{CH}-4 \mathrm{~T} / \mathrm{HSA}-\mathrm{HT}$ were injected sequentially into both footpads at an exposure time of 300 and $400 \mathrm{~ms}$, respectively. ICG and CH-4T imaging performed with a silicon and InGaAs camera, respectively, with identical $785 \mathrm{~nm}$ excitation laser conditions. Colourbar spans 0-1,500 for ICG and 0-8,000 for $\mathrm{CH}-4 \mathrm{~T} / \mathrm{HSA}-\mathrm{HT}$. Scale bars, $1 \mathrm{~cm}$. (g). White arrows correspond to direction of the $\mathbf{g}$ fluorescent cross-sectional intensity profile of lumbar lymph nodes on each detector.

with the lowest exposures time to date for imaging at NIR-II wavelengths ${ }^{1}$. Small changes in the molecular structure of $\mathrm{CH} 1055$ yielded a dramatic increase in the optical performance of the D-A-D dye, while further optimization of the electron acceptor, $\pi$-spacers, electron donors and functional groups is likely to further increase the maximum attainable NIR-II quantum yield.

The advent of a high quantum yield NIR-II fluorophore enables many exciting possibilities for NIR-II medical imaging in terms of exposure time, imaging speed and penetration depth. Carbon nanotubes, encapsulated organic dyes and $\mathrm{Ag}_{2} \mathrm{~S}$ quantum dot contrast agents have required exposure times of $100 \mathrm{~ms}$ and may even exceed $200 \mathrm{~ms}$ for video-rate imaging ${ }^{2-5}$. The instrument overhead time for normal video-rate imaging is $\sim 80 \mathrm{~ms}$ and results in a frame rate of 5.3 FPS at a typical NIR-II exposure time of $100 \mathrm{~ms}$. The donor-acceptor NIR-II polymer with a nominal $1.4 \%$ quantum yield allowed ultra-fast videos at $\sim 20 \mathrm{~ms}$ exposure times and frame rates of $25 \mathrm{FPS}^{1}$. The $\mathrm{CH}-4 \mathrm{~T}$ complex was able to easily capture ultra-fast videos under $1.5-2 \mathrm{~ms}$ exposure times at 50 FPS, which is virtually at the maximum possible frame rate of our imaging system (52 FPS) given the instrument overhead time of $19 \mathrm{~ms}$ under ultra-fast imaging conditions.

Even though $\mathrm{CH}-4 \mathrm{~T}$ has a significant higher brightness than the NIR-II polymer, the achievable frame rate with $\mathrm{CH}-4 \mathrm{~T}$ is only double that of the polymer as the instrument overhead time is now the main factor limiting the time in-between frames ${ }^{1}$.
However, designing faster fluorescent NIR-II imaging systems through hardware and software modifications could theoretically yield frame rates surpassing 500 FPS given the 1.5-2 ms exposure time of the CH-4T complexes. Such a high-brightness dye additionally allows the detection of deeper features in the body as evidenced by the clear resolution of the lumbar lymph nodes. As the excitation power density drops off when penetrating deeper into the body, high quantum yield NIR-II fluorophores are necessary to generate sufficient fluorescent signal. Although previous materials such as carbon nanotubes or $\mathrm{CH}-\mathrm{PEG}$ can clearly discern more shallow features with a high degree of clarity due to minimal scattering, they have an insufficient quantum yield to detect deep features (Supplementary Fig. 13). The high quantum yield NIR-II dye also lowers the requisite excitation power density as evidenced by the imaging of the lumbar lymph nodes with ICG and CH-4T/HSA-HT at an equivalent power density of $16 \mathrm{~mW} \mathrm{~cm}^{-2}$ and with similar exposure times. Previously, a 17.5-fold higher power density was required for imaging femoral blood vasculature in the hindlimb with carbon nanotubes in the NIR-II (808 $\mathrm{nm}$ excitation, $140 \mathrm{~mW} \mathrm{~cm}^{-2}$ ) compared to IR800 in the NIR-I $(785 \mathrm{~nm}$ excitation, $\left.8 \mathrm{~mW} \mathrm{~cm}^{-2}\right)^{4}$.

At this juncture, we would like to highlight the variability in the reported absolute quantum yield of IR-26, the reference fluorophore that serves as the basis for all reported NIR-II quantum yields. The first reported quantum yield of IR-26 as $0.5 \%$ in 1,2 -dichloroethane (DCE), the most widely applied value, 
has been called into question by recent reports placing the quantum at both 0.05 and $0.1 \%$ (refs 31-34). The reported quantum yield for $\mathrm{CH}-4 \mathrm{~T}$ and complexes is based on using HiPCO SWCNTs as a reference, whose quantum yield was similar to IR-26 (ref. 1). HiPCO SWCNTs were selected as a quantum yield reference as nanotubes are simple to disperse in water, demonstrate excellent stability unlike IR-26 that degrades in 1,2-dichloroethane over relatively short periods to time, and are the most common NIR-II contrast agent ${ }^{5}$. The brightness of NIR-II dyes will be listed relative to other NIR-II fluorophores as well as in absolute quantum yield ranges henceforth until the discrepancies in the reported quantum yield of IR-26 are resolved. As this work marks a transition in the reference absolute quantum yield, the absolute quantum yield of $\mathrm{CH}-4 \mathrm{~T}$ is $1.1-11 \%$ with respect to IR-26 $=0.05-0.5 \%$. Changing the absolute quantum yield of the reference only serves to scale the brightness of the entire NIR-II field and does not affect the relative brightness between NIR-II emitting materials. Regardless, $\mathrm{CH}-4 \mathrm{~T}$ is by far the brightest organic fluorophore of any NIR-I or NIR-II dye emitting past $1,000 \mathrm{~nm}$ (Supplementary Fig. 14) with a 27-fold higher quantum yield than HiPCO SWCNTs, a classic NIR-II imaging probe.

Tuning the interactions between organic NIR-II dyes consisting of large, hydrophobic $\pi$-systems and plasma proteins is a route to brilliantly fluorescent dyes emitting past $1,000 \mathrm{~nm}$. In a similar fashion to ICG's $\sim 4 \times$ increase in brightness when exposed to serum proteins, the 110-fold fluorescence increase between $\mathrm{CH}-4 \mathrm{~T} / \mathrm{FBS}-\mathrm{HT}$ and $\mathrm{CH}-4 \mathrm{~T}$ in PBS most likely occurs through the combination of multiple processes ${ }^{35,36}$. First, the amphiphilic character of $\mathrm{CH}-4 \mathrm{~T}$ may form aggregates in aqueous solution through van der Waals forces that reduce brightness through self-quenching which is known to occur in $\mathrm{ICG}^{29}$. The dispersion of aggregates by $\mathrm{CH}-4 \mathrm{~T}$ individualization through protein binding should contribute to the serum boost in quantum yield. Second, $\mathrm{CH}-4 \mathrm{~T}$ geometrical confinement by hydrophobic pockets should promote a rigid dye conformation that minimizes the torsional rotations that result in non-radiative decay ${ }^{12}$. Rigid molecules have shorter fluorescent lifetimes and generally higher quantum yields as they dissipate less energy though internal conversion compared to those with freely rotating parts ${ }^{37}$. Internal conversion is the most important competing nonemissive process for low energy-gap NIR-II dyes thus increasing rigidity through protein binding will favourably increase quantum yield. Heating magnifies these effects as $\mathrm{CH}-4 \mathrm{~T}$ presumably becoming entangled in a rigid conformation as increased thermal energy allows access to typically inaccessible hydrophobic pockets. At higher temperatures, $\mathrm{CH}-4 \mathrm{~T}$ may become completely encased in hydrophobic domains as proteins re-fold around the dye after heating to minimize the number of hydrophobic side-chains exposed to water. In addition, donoracceptor dyes are subject to twisted intramolecular charge transfer (TICT) in polar environments, especially if comprised of strong electron donors and acceptors ${ }^{38-41}$. Molecular simulations predict the core of $\mathrm{CH} 1055$ can exist in non-planar conformations that may contribute to a red-shifted TICT conformation that can dampen fluorescence ${ }^{12}$. The observed hypsochromic shift of $\sim 50 \mathrm{~nm}$ can be explained through $\mathrm{CH}-4 \mathrm{~T}$ adopting a planar configuration and the alignment of $p$-orbitals when binding to proteins via hydrophobic or charge interactions in conjunction with minimizing solvent relaxation.

Developing a fundamental understanding of the manner in which NIR-II organic dyes interact with biological tissue is of critical importance for creating probes with high SBR ratios as well as high excretion levels. $\mathrm{CH}-4 \mathrm{~T}$ demonstrated an impressively high quantum yield thorough protein binding and the dye was excreted through the biliary system with little residual dye left in normal tissue just days post-injection. Unlike current NIR-II nanomaterial fluorophores, $\mathrm{CH}-4 \mathrm{~T}$ demonstrated complete excretion from the body, albeit at a slower rate than $\mathrm{CH}-\mathrm{PEG}$ (Supplementary Fig. 15). CH-4T elicited no observable cellular toxicity (Supplementary Figs 16 and 17), while CH-4T/FBS-HT and $\mathrm{CH}-4 \mathrm{~T} / \mathrm{HSA}-\mathrm{HT}$ showed a marginal reduction in cell viability at higher concentrations (Supplementary Figs 18 and 19). However, in a similar manner to the pioneering work by Frangioni and colleagues, tailoring the interaction strength between NIR-II fluorophores and proteins to maintain a high quantum yield while employing strategies such as polyionic yet net-neutral probes may expedite excretion kinetics and reduce non-specific in vivo binding ${ }^{42,43}$. The same rational design principles developed for NIR-I fluorophores may be applied to NIR-II organic dyes to develop the next generation of contrast and molecular imaging agents.

Hemodynamic imaging with a high frame rate in the NIR-II poses several advantages over other vascular flow imaging modalities such as laser speckle flowgraphy and photoacoustic imaging (PI). Laser speckle flowgraphy computes blood velocity based on the measurement of laser speckle blurring (termed normalize blur (NB)) from coherent light scattering from diffusing sources ${ }^{44,45}$. Modulation of the laser backscattered interference pattern occurs as red blood cells travel through capillaries. Laser speckle flowgraphy has significant clinical potential as it requires no exogenous contrast agents yet this imaging modality currently suffers from a number limitations. Measurement of laser flux depends highly on tissue properties that can vary from person to person or in disparate disease states. A correlation between the unit-less NB and capillary blood flow velocities occurs only under a narrow set of conditions ${ }^{44,45}$. This disallows facile blood velocity comparisons among people or even between an individual's eyes during retinal vasculature imaging. Photoacoustic imaging can additionally measure blood velocity without requiring exogenous contrast agents by exploiting hemoglobin's strong visible absorbance. However, with minimum resolvable features on the scale of $\sim 10-100 \mu \mathrm{m}$, photoacoustic imaging cannot compete with fluorescence imaging in terms of spatial resolutionx ${ }^{46}$. CH-4T's penetration depth (1-3 mm) combined with high temporal (50 FPS) and spatial $(\sim 1 \mu \mathrm{m})$ resolution enables high-fidelity hemodynamic imaging of vessel sizes ranging from microvasculature to major vessels. Application of high frame-rate NIR-II imaging with $\mathrm{CH}$ 4T for hemodynamic visualization within tumours, intravascular tracking of circulating tumour cells or post-trauma cerebral microvascular imaging may provide key insight into disease pathophysiology. In addition to providing absolute blood velocities, CH-4T's high-brightness and long-wavelength emission may improve other biological optical imaging modalities. For instance, one-photon confocal imaging with $\mathrm{CH}-4 \mathrm{~T}$ may push the penetration depth limit past that achievable with the current palette of visible/NIR-I/NIR-II fluorophores.

Even with the superior NIR-I silicon CCD detector specifications (for example, pixel number, dark current, readout noise and so on), NIR-II imaging on the InGaAs detector with longer wavelength probes improves imaging metrics. As no current silicon detector has suitable sensitivity past $\sim 1,000 \mathrm{~nm}$, biomedical fluorescent imaging in the NIR-II depended on the emergence of InGaAs focal plane array detectors. However, similarly to the benefits garnered by pushing probe emission out of the visible $(\sim 350-600 \mathrm{~nm})$ and into the NIR-I $(\sim 650-900 \mathrm{~nm})$ on $\mathrm{Si}$ cameras, the development of bright, long-wavelength NIR-II probes $(>1,000 \mathrm{~nm})$ boosts imaging performance metrics. Internal comparisons within a broad wavelength range on an InGaAs detector have dramatically illustrated the imaging improvements by developing longer 
wavelength probes from $850 \mathrm{~nm}$ (ICG) to $1,100 \mathrm{~nm}$ (HiPCO SWCNTs) to $1,500 \mathrm{~nm}$ (laser vaporization SWCNTs) ${ }^{14}$. ICG and laser vaporization SWCNTs both emit in a region with similarly reduced InGaAs quantum efficiency of $\sim 30-40 \%$ compared to $\sim 85 \%$ at $\sim 1,100 \mathrm{~nm}$ with HiPCO SWCNTs. This indicates the marked differences in imaging quality at progressively longer wavelengths arise from reduced scattering/autofluorescence rather than variable detector spectral sensitivities. Fundamental advances in fluorophore chemistry have largely driven the NIR-II imaging field, making InGaAs detectors more commonplace for biomedical imaging. While newer generations of InGaAs detectors can uniformly increase sensitivity/SBR of all NIR-II probes, relative imaging metrics within the NIR-II spectral range will still be governed by wavelength dependent light-tissue interactions. Improving NIR-II fluorescent probes (that is, increasing both quantum yield and NIR-II emission wavelength) should proceed in parallel with the ongoing innovations in improving InGaAs cameras.

In summary, we have developed the first NIR-II contrast agent based on a small-molecule organic dye with an ultra-high quantum yield of $1.1-11 \%$ (IR-26=0.05-0.5\%). The negatively charged sulfonate groups enabled supramolecular binding to serum proteins capable of a 110 -fold fluorescence enhancement compared to $\mathrm{CH}-4 \mathrm{~T}$ in PBS. Heating the $\mathrm{CH}-4 \mathrm{~T}-$ protein complex caused a marked boost in fluorescence intensity indicating that brightness increases are possible through optimizing dye-protein interactions. In vivo vascular imaging was performed at 50 FPS, the fastest frame rate to date in the NIR-II at an exposure time of $2 \mathrm{~ms}$ to unambiguously resolve vascular hemodynamics. In addition, imaging of deep lymph nodes at a depth of $\sim 5-8 \mathrm{~mm}$ in both the first and second NIR windows clearly demonstrated the benefits of imaging deep anatomical features at longer wavelengths. $\mathrm{CH}-4 \mathrm{~T}$ illustrates for the first time that high quantum yield dyes in the NIR-II are possible. Future work will strive to modify $\mathrm{CH}-4 \mathrm{~T}$ to enable covalent linkable NIR-II fluorophores whose brightness can be utilized for ultra-high SBR in vivo molecular imaging.

\begin{abstract}
Methods
Animal handling. All vertebrate animal experiments were performed under the approval of Stanford University's Administrative Panel on Laboratory Animal Care. Eight-week-old female NU/NU mice were obtained from Charles River for all imaging studies and housed at the Research Animal Facility of Stanford under our approved animal protocols. Before vessel or lymphatic imaging, all mice were anaesthetized in a rodent anaesthesia machine with $21 \mathrm{~min}^{-1} \mathrm{O}_{2}$ gas mixed with $3 \%$ isoflurane. Tail vein injection of contrast agents were carried with a catheter and synchronized with a camera that started continuous image acquisition simultaneously. The injected dose was a $200-300 \mu \mathrm{l}$ bolus in a $1 \times$ PBS solution at specified concentrations. During the time course of imaging the mouse was kept anaesthetized by a nose cone delivering $21 \mathrm{~min}^{-1} \mathrm{O}_{2}$ gas mixed with $3 \%$ isoflurane. The sample sizes of mice were selected based on previously reported studies. Mice were randomly selected from cages for all experiments. No blinding was performed. All groups within study contained $n=3$ mice.
\end{abstract}

Synthesis of CH-4T. Dissolve $1 \mathrm{mg}$ CH1055 $(1.03 \mu \mathrm{mol})$ in $100 \mu \mathrm{l}$ of dry DMSO. Then $5 \mathrm{mg}$ taurine $(40 \mu \mathrm{mol}), 7 \mu \mathrm{l}$ DIPEA $(5.17 \mathrm{mg}, 40 \mu \mathrm{mol})$ were added. Stirred for $2 \mathrm{~min}$ and then add HBTU $5 \mathrm{mg}(13.2 \mu \mathrm{mol})$. The reaction solution was stirred overnight at room temperature under a nitrogen atmosphere. After the reaction finished, $100 \mu \mathrm{l}$ of water was added and stirred for $1 \mathrm{hr}$ to quench excess HBTU. Finally, Dionex Summit high-performance liquid chromatography (HPLC) system (Dionex Corporation, Sunnyvale, CA, USA), 340U four-channel ultraviolet-visible absorbance detector, Dionex C4, $9.4 \mathrm{~mm} \times 250 \mathrm{~mm}$ semi-preparative column, gradient elution starting from $5 \%$ acetonitrile and ending up with $95 \%$ acetonitrile (in water with $0.1 \%$ TFA) at $42 \mathrm{~min}, 3 \mathrm{ml} \mathrm{min}^{-1}$ flow rate, $254 \mathrm{~nm}$ and $650 \mathrm{~nm}$ detection wavelength was used to purify the reaction. Overall, $1.3 \mathrm{mg} \mathrm{CH} 1055-$ 4Taurine (yield 93\%) was produced as a green solid. MALDI-TOF-MS was used to identify the product. MALDI-TOF-MS calculated for: $\left[\mathrm{C}_{62} \mathrm{H}_{64} \mathrm{~N}_{10} \mathrm{O}_{16} \mathrm{~S}_{6}\right]$ (M.W.): 1,396.3. Found: $1,396.2$. See Supplementary Information for additional details.

DGU of CH-4T and CH-4T complexes. Sucrose density gradients were prepared in $4 \mathrm{ml}$ centrifuge tube. A total of $600 \mu \mathrm{l}$ of $55,50,45,40,35,30 \% \mathrm{w} / \mathrm{w}$ sucrose in
$1 \times$ PBS were added through a syringe with a needled pressed against centrifuge tube wall to not cause layer mixing. After adding all density layers, the centrifuge tube was placed at $10^{\circ}$ from horizontal to allow for interlayer diffusion to smooth out density steps. After $1 \mathrm{~h}, \mathrm{CH}-4 \mathrm{~T}$ samples were loaded on top of the gradient. Any excess space in centrifuge tube was filled with $1 \times$ PBS until meniscus reached $\sim 3 \mathrm{~mm}$ from top of centrifuge tube. The gradient was run in an ultracentrifuge (Beckman Coulter, Optima L-90K) using a Ti-55 rotor at 50,000 r.p.m. for $50 \mathrm{~h}$.

Preparation of CH-4T heated complexes. Prepared $784 \mu \mathrm{M} \mathrm{CH}-4 \mathrm{~T}, 78.4 \mu \mathrm{M}$ HSA/BSA in $1 \times$ PBS solution. Added $10 \mu \mathrm{l}$ of $784 \mu \mathrm{M}$ CH- $4 \mathrm{~T}$ into $200 \mu \mathrm{l}$ of $78.4 \mu \mathrm{M}$ HSA/BSA solution to keep the molar ratio of CH-4T:HSA at 1:2 for optical characterization (1:1 ratio for in vivo HSA imaging). (HSA: Sigma Aldrich, Lot\# SLBN5035V, SLBL6442V; BSA: Sigma Aldrich, Lot\# SLBR6762V). Keep overall protein concentration as low as possible to prevent possible gelling after heating. For FBS, use maximum dye loading of $50 \mu \mathrm{g}$ CH-4T/100 $\mu \mathrm{l} \mathrm{FBS}$ (see Supplementary Fig. 4 for manufacturer/batches). Vortex the solution to mix evenly. Then add the dye-protein complex in a sealed eppendorf into different temperature water baths for $10 \mathrm{~min}\left(\sim 70-75^{\circ} \mathrm{C}\right.$ for in vivo complexes). Discard if solution gels or denatures by turning white. Temperatures kept under $80^{\circ} \mathrm{C}$ should not gel. If not used within a few hours, store the heated dye-protein solution at $4{ }^{\circ} \mathrm{C}$ for long-term storage. $\mathrm{CH}$-PEG heated complexes were prepared by the same procedure for comparison.

Spectral characterization of $\mathbf{C H}-\mathbf{4 T}$. Absorbance spectra of $\mathrm{CH}-4 \mathrm{~T}$ and complexes were taken on a ultraviolet-visible-NIR Cary 6000i spectrometer that was background corrected for each biological media such as water, FBS and HSA protein solutions. The NIR-II fluorescence emission spectrum was captured on a home-built spectroscopy set-up by exciting $\mathrm{CH}-4 \mathrm{~T}$ and complexes with an $808 \mathrm{~nm}$ laser diode with a power output of $\sim 160 \mathrm{~mW}$. The excitation laser was filtered with a combination of an 850 (Thorlabs)/1,000 (Thorlabs)/1,100 (Omega)/1,200 (Thorlabs)/1,300 (Omega) nm short-pass filters. Samples were added to either a $1 \mathrm{~mm}$ or $1 \mathrm{~cm}$ path-length cuvette and the resulting emission filtered through a $910 \mathrm{~nm}$ long-pass filter (Thorlabs) to reject the incident excitation laser light. The emitted fluorescence was collected on a spectrometer (Acton SP2300i) coupled to a linear liquid nitrogen cooled InGaAs detector array (Princeton Instruments, OMA-V). After collecting the raw acquisition data, a correction file was applied to correct for the variable InGaAs quantum efficiency as a function of detection wavelength as well as the variable $910 \mathrm{~nm}$ long-pass filter extinction features across the NIR-II spectral region. Fluorescence enhancement defied as:
$\left(\int_{900}^{1,500} I_{\mathrm{CH} 4 \mathrm{~T} / \mathrm{X}} d_{\lambda}\right) /\left(\int_{900}^{1,500} I_{\mathrm{CH} 4 \mathrm{~T} / \mathrm{PBS}} d_{\lambda}\right)$, where $I_{\mathrm{CH}-4 \mathrm{~T} / \mathrm{X} \text { corresponds to the }}$ fluorescent emission spectrum of protein-bound $\mathrm{CH}-4 \mathrm{~T}$. All fluorescent enhancement values were derived from measurements on the wavelength-corrected photospectrometer unless specifically stated otherwise.

Measuring NIR-II quantum yield. The fluorescence quantum yield of $\mathrm{CH}-4 \mathrm{~T}$ and complexes were measured in a similar manner as described in previous publications ${ }^{1,2}$. Briefly, HiPCO SWCNTs were utilized as a reference fluorophore due to their high aqueous stability. The quantum yield of HiPCO SWCNTs has previously been determined as $0.4 \%$ based on an IR-26 quantum yield of $0.5 \%$ in DCE (please see Discussion for further details on IR-26 quantum yield). A serial dilution of five solutions of HiPCO SWCNTs as well as CH-4T and complexes with an OD $<0.1$ at $808 \mathrm{~nm}$ was measured to confirm absorbance values at $808 \mathrm{~nm}$ and the fluorescent emission spectrum was collected on a wavelength-corrected NIR-II spectrometer in a $1 \mathrm{~cm}$ quartz cuvette (Starna) in the manner specified above. The fluorescent emission spectrum was integrated and plotted against the $\mathrm{OD}$ value at $808 \mathrm{~nm}$ and a linear fit was applied to verify the linearity between fluorescent brightness and concentration. For the brightest samples, inter-filter effects were seen $\sim$ OD 0.1 at $808 \mathrm{~nm}$, thus lower concentration ranges were utilized. By comparing the slope of the linear fit between HiPCO SWCNTs and CH-4T and complexes, the quantum yield was determined based on the following supporting equation (1):

$$
\mathrm{QY}_{\text {sample }}=\mathrm{QY}_{\text {ref }} \times \frac{\text { slope }_{\text {sample }}}{\text { slope }_{\text {ref }}} \times\left(\frac{n_{\text {sample }}}{n_{\text {ref }}}\right)^{2}
$$

where $\mathrm{QY}_{\text {sample }}$ is the $\mathrm{QY}$ of $\mathrm{CH}-4 \mathrm{~T}$ and complexes, $\mathrm{QY}$ ref is the QY of HiPCO SWCNTs in water $\left(0.4 \%\right.$ based on IR-26 $=0.5 \%$ in DCE $\left.{ }^{1}\right), n_{\text {sample }}$ and $n_{\text {ref }}$ are the refractive indices of HiPCO SWCNTs, $\mathrm{CH}-4 \mathrm{~T}$ and complex solutions which are both water (1.33) in this case.

Video-rate and ultra-fast NIR-II imaging. Video-rate and ultra-fast NIR-II imaging was carried out in a similar manner as previous publication ${ }^{1,2}$. Briefly, all NIR-II images were collected on a $319 \times 256$ pixel two-dimensional InGaAs array (Princeton Instruments 2D OMA-V 1.7; pixel number: $319 \times 256$; readout noise: 50 electron r.m.s.; dark signal: 5,000 electrons per second per pixel) utilizing a home-built imaging set-up. The excitation was provided by an $808 \mathrm{~nm}$ diode laser (RMPPC Lasers) through an optical fibre and collimator at a power density of $140 \mathrm{~mW} \mathrm{~cm}^{-2}$. The $808 \mathrm{~nm}$ laser was filtered with $850 / 1,000 \mathrm{~nm}$ short-pass filters (Thorlabs). Fluorescence emission was collected with a $910 \mathrm{~nm}$ long-pass filter 
(Thorlabs) combined with either a 1,000 nm long-pass filter (Thorlabs) or 1,100 nm long-pass filter (Thorlabs) as specified. A ' $\times 2.5$ ' magnification lens set (' $\mathrm{C}$ ' coated, $200 \mathrm{~mm}$ and $75 \mathrm{~mm}$, Thorlabs) was used for the mice hindlimb NIR-II imaging. Changing the relative position of the two NIR achromats can modify the magnifications into ' $\times 1$ ' for whole body imaging. For ultra-fast imaging, the camera is set to continuously expose using LabView software with a 19-ms overhead time caused by camera readout. Images and videos were processed with MATLAB. Imaging the mouse in a supine position with the leg and chest region well secured on the imaging platform with medical tape prevented any motion artifacts resulting from breathing motion or heartbeat. Selecting a $3 \times 3$ pixel region corresponding to the femoral artery in each video frame allowed for analysis of the fluorescent intensity in hindlimb vasculature. The femoral vasculature mapped to the same pixel group throughout imaging, indicating that the leg is secure and that the periodic patterns do not arise from breathing induced artifacts. Please see Supplementary Sections 12 and 13 for detailed schematic of optical path with component product information and catalogue numbers.

Sacral/popliteal and lumbar lymph node NIR-I/II imaging. Sacral/popliteal and lumbar lymph node NIR- II imaging was performed using the whole body set-up as described in the above paragraph. A 1,344 × 1,024-pixel Hamamatsu silicon CCD detector (Hamamatsu C8484-03G02; pixel number: 1,344 × 1,024; readout noise: $\sim 7$ electron r.m.s.; dark current: 0.01 electrons per second per pixel) with a $785 \mathrm{~nm}$ long-pass filter (Semrock) for NIR-I imaging was added in parallel to the NIR-II set-up for NIR-I imaging comparisons as described in previous publications ${ }^{4}$. The $785 \mathrm{~nm}$ laser (filtered with $780 \mathrm{~nm}$ band-pass filter) was used both for NIR-I and NIR-II imaging with a power density of $16 \mathrm{~mW} \mathrm{~cm}^{-2}$. All contrast agents (ICG, CH-4T, CH-4T/HSA-HT) were injected into each digit and the footpad under anaesthesia. Mice were then immediately placed on the imaging platform. A gentle massage was performed on the injection site for faster diffusion into the lymphatic system. For deep lumbar lymph node imaging, ICG was first injected as described above. The mice were taped securely in a position where the lumbar lymph nodes were visualized on the silicon NIR-I detector. $\mathrm{CH}-4 \mathrm{~T}$ complexes were subsequently injected $\sim 10$ min later for imaging in the NIR-II. Fluorescent intensity cross-sectional profiles in the NIR-I versus NIR-II were analysed in Image J. Please see Supplementary Sections 12 and 13 for detailed schematic of optical path with component product information and catalogue numbers.

Data availability. Data supporting the findings of this study are available within the article (and its Supplementary Information files) and from the corresponding author upon reasonable request.

\section{References}

1. Hong, G. et al. Ultrafast fluorescence imaging in vivo with conjugated polymer fluorophores in the second near-infrared window. Nat. Commun. 5, 4206 (2014).

2. Antaris, A. L. et al. A small-molecule dye for NIR-II imaging. Nat. Mater. 15, 235-242 (2016).

3. Hong, G. et al. Through-skull fluorescence imaging of the brain in a new nearinfrared window. Nat. Photonics 8, 723-730 (2014).

4. Hong, G. et al. Multifunctional in vivo vascular imaging using near-infrared II fluorescence. Nat. Med. 18, 1841-1846 (2012).

5. Welsher, K. et al. A route to brightly fluorescent carbon nanotubes for near-infrared imaging in mice. Nat. Nanotechnol. 4, 773-780 (2009).

6. Yi, H. et al. M13 phage-functionalized single-walled carbon nanotubes as nanoprobes for second near-infrared window fluorescence imaging of targeted tumors. Nano Lett. 12, 1176-1183 (2012).

7. Dang, X. et al. Layer-by-layer assembled fluorescent probes in the second nearinfrared window for systemic delivery and detection of ovarian cancer. Proc. Natl Acad. Sci. USA 113, 5179-5184 (2016).

8. Sordillo, L. A., Pu, Y., Pratavieira, S., Budansky, Y. \& Alfanoa, R. R. Deep optical imaging of tissue using the second and third near-infrared spectral windows. J. Biomed. Opt. 19, 056004 (2014).

9. Hong, G. et al. Near-Infrared II fluorescence for imaging hindlimb vessel regeneration with dynamic tissue perfusion measurement. Circ. Cardiovasc. Imaging 7, 517-525 (2014).

10. Zhang, X.-D. et al. Traumatic brain injury imaging in the second near-infrared window with a molecular fluorophore. Adv. Mater. 28, 6872-6879 (2016).

11. Sun, Y. et al. Novel benzo-bis(1,2,5-thiadiazole) fluorophores for in vivo NIR-II optical imaging. Chem. Sci. 7, 6203-6207 (2016).

12. Hof, M., Hutterer, R. \& Fidler, V. Fluorescence Spectroscopy in Biology: advanced Methods and Their Applications to Membranes, Proteins, DNA, and Cells (Springer, 2005).

13. Umezawa, K., Nakamura, Y., Makino, H., Citterio, D. \& Suzuki, K. Bright, color-tunable fluorescent dyes in the visible-near-infrared region. J. Am. Chem. Soc. 130, 1550-1551 (2008).

14. Diao, S. et al. Fluorescence imaging in vivo at wavelengths beyond $1500 \mathrm{~nm}$. Angew. Chem. Int. Ed. 54, 14758-14762 (2015).
15. Chen, J. et al. Direct water-phase synthesis of lead sulfide quantum dots encapsulated by $\beta$-lactoglobulin for in vivo second near infrared window imaging with reduced toxicity. Chem. Commun. 52, 4025-4028 (2016).

16. Kong, Y. et al. Highly fluorescent ribonuclease-A-encapsulated lead sulfide quantum dots for ultrasensitive fluorescence in vivo imaging in the second near-infrared window. Chem. Mater. 28, 3041-3050 (2016).

17. Qian, G. et al. Band gap tunable, donor - acceptor - donor charge-transfer heteroquinoid-based chromophores: near infrared photoluminescence and electroluminescence. Chem. Mater. 20, 6208-6216 (2008).

18. Bhasikuttan, A. C., Mohanty, J., Nau, W. M. \& Pal, H. Efficient fluorescence enhancement and cooperative binding of an organic dye in a supra-biomolecular host-protein assembly. Angew. Chem. Int. Ed. 46, 4120-4122 (2007).

19. Megyesi, M. \& Biczok, L. Considerable fluorescence enhancement upon supramolecular complex formation between berberine and p-sulfonated calixarenes. Chem. Phys. Lett. 424, 71-76 (2006).

20. Ifft, J. B. Sedimentation equilibrium of proteins in density gradients. Biophys. Chem. 5, 137-157 (1976)

21. Hutteman, M. et al. Optimization of near-infrared fluorescent sentinel lymph node mapping for vulvar cancer. Am. J. Obstet. Gynecol. 206, 89.e1-5 (2012).

22. Bardhan, N. M., Ghosh, D. \& Belcher, A. M. Carbon nanotubes as in vivo bacterial probes. Nat. Commun. 5, 4918 (2014).

23. Iverson, N. M. et al. In vivo biosensing via tissue-localizable near-infraredfluorescent single-walled carbon nanotubes. Nat. Nanotechnol. 8, 873-880 (2013).

24. Yi, H. J. et al. M13 phage-functionalized single-walled carbon nanotubes as nanoprobes for second near-infrared window fluorescence imaging of targeted tumors. Nano Lett. 12, 1176-1183 (2012).

25 . Diao, S. et al. Chirality enriched $(12,1)$ and $(11,3)$ single-walled carbon nanotubes for biological imaging. J. Am. Chem. Soc. 134, 16971-16974 (2012).

26. Ghosh, D. et al. Deep noninvasive imaging and surgical guidance of submillimeter tumors using targeted M13-stabilized single-walled carbon nanotubes. Proc. Natl Acad. Sci. USA 111, 13948-13953 (2014).

27. Tao, Z. M. et al. Biological Imaging using nanoparticles of small organic molecules with fluorescence emission at wavelengths longer than $1000 \mathrm{~nm}$. Angew. Chem. Int. Ed. 52, 13002-13006 (2013).

28. Hutteman, M. et al. Randomized, double-blind comparison of indocyanine green with or without albumin premixing for near-infrared fluorescence imaging of sentinel lymph nodes in breast cancer patients. Breast Cancer Res. Treat. 127, 163-170 (2011)

29. Desmettre, T., Devoisselle, J. M. \& Mordon, S. Fluorescence properties and metabolic features of indocyanine green (ICG) as related to angiography. Surv Ophthalmol. 45, 15-27.

30. Kirchher, A.-M., Briel, A. \& Mader, K. Stabilization of indocyanine green by encapsulation within micellar systems. Mol. Pharm. 6, 480-491 (2009).

31. Murphy, J. E. et al. PbTe colloidal nanocrystals: synthesis, characterization, and multiple exciton generation. J. Am. Chem. Soc. 128, 3241-3247 (2006).

32. Kopainsky, B., Qiu, P., Kaiser, W., Sens, B. \& Drexhage, K. H. Lifetime, photostability, and chemical structure of IR heptamethine cyanine dyes absorbing beyond $1 \mu \mathrm{m}$. Appl. Phys. B 29, 15-18 (1982).

33. Semonin, O. E. et al. Absolute photoluminescence quantum yields of IR-26 dye, PbS, and PbSe quantum dots. J. Phys. Chem. Lett. 1, 2445-2450 (2010).

34. Hatami, S. et al. Absolute photoluminescence quantum yields of IR26 and IRemissive $\mathrm{Cd}_{1-x} \mathrm{Hg}_{x} \mathrm{Te}$ and $\mathrm{PbS}$ quantum dots -method- and material-inherent challenges. Nanoscale 7, 133-143 (2015).

35. Benson, R. C. \& Kues, H. A. Fluorescence properties of indocyanine green as related to angiography. Phys. Med. Biol. 23, 159-163 (1978).

36. Mordon, S., Devoisselle, J. M., Soulie-Begu, S. \& Desmettre, T. Indocyanine green: physicochemical factors affecting its fluorescence in vivo. Microvasc. Res 55, 146-152 (1998)

37. Siegel, J. A. Principles of fluorescence spectroscopy. Choice Curr. Rev. Acad. Lib. 44, 1196-1196 (2007).

38. Reja, S. I., Khan, I. A., Bhalla, V. \& Kumar, M. A TICT based NIR-fluorescent probe for human serum albumin: a pre-clinical diagnosis in blood serum. Chem. Commun. 52, 1182-1185 (2016).

39. Sasaki, S., Drummen, G. P. C. \& Konishi, G. Recent advances in twisted intramolecular charge transfer (TICT) fluorescence and related phenomena in materials chemistry. J. Mater. Chem. C 4, 2731-2743 (2016).

40. Rettig, W. Photophysical and photochemical switches based on twisted intramolecular charge transfer (TICT) states. Appl. Phys. B 45, 145-149 (1988).

41. Cao, C. et al. A twisted-intramolecular-charge-transfer (TICT) based ratiometric fluorescent thermometer with a mega-Stokes shift and a positive temperature coefficient. Chem. Commun. 50, 15811-15814 (2014).

42. Choi, H. S. et al. Targeted zwitterionic near-infrared fluorophores for improved optical imaging. Nat. Biotechnol. 31, 148-153 (2013).

43. Choi, H. S. et al. Synthesis and in vivo fate of zwitterionic near-infrared fluorophores. Angew. Chem. Int. Ed. 50, 6258-6263 (2011).

44. Senarathna, J., Rege, A., Li, N. \& Thakor, N. V. Laser speckle contrast imaging: theory, instrumentation and applications. IEEE Rev. Biomed. Eng. 6, 99-110 (2013). 
45. Sugiyama, T., Araie, M., Riva, C. E., Schmetterer, L. \& Orgul, S. Use of laser speckle flowgraphy in ocular blood flow research. Acta Ophthalmol. 88, 723-729 (2010).

46. Hu, S. \& Wang, L. V. Photoacoustic imaging and characterization of the microvasculature. J. Biomed. Opt. 15, 011101 (2010).

\section{Acknowledgements}

This work was partially supported by the Office of Science (BER), US Department of Energy (DE-SC0008397) (Z.C.), NCI Cancer Center Nanotechnology Excellence Gran (CCNE-TR) U54 CA119367, the Calbrain Programme, a Neurotechnology Programme of California (H.D.), CA151459, the National Natural Science Foundation of China 81573383, 81373254 and 21390402, NSFHP (2014CFB704) (X.H.), International S\&T Cooperation Programme of China (2015DFA30440, 2014DFB30020) (X.H.), the National Science and Technology Major Project of the Ministry of Science and Technology of China No. 2012ZX10004801-003-011 (X.H.), Key Project of Chinese Ministry of Education No. 313040 (X.H.), Academic Award for Excellent PhD Candidates funded by Ministry of Education of China (No. 5052012306001), the Fundamental Research Funds for the Central Universities (C.Q., H.C., X.H.) and Innovation Seed Fund of Wuhan University School of Medicine (X.H.).

\section{Author contributions}

Z.C., H.D. and X.H. conceived and designed the study, supervised the project and wrote the manuscript. A.L.A. and H.C. designed the study, performed all the experiments and wrote the manuscript. S.D., Z.M. helped with infrared. S.Z., L.C., J.W., A.X.L. helped prepared dye-protein complexes as well as perform optical characterization.

\section{Additional information}

Supplementary Information accompanies this paper at http://www.nature.com/ naturecommunications

Competing interests: The authors declare no competing financial interests.

Reprints and permission information is available online at http://npg.nature.com/ reprintsandpermissions/

How to cite this article: Antaris, A. L. et al. A high quantum yield molecule-protein complex fluorophore for near-infrared II imaging. Nat. Commun. 8, 15269 doi: 10.1038/ ncomms15269 (2017).

Publisher's note: Springer Nature remains neutral with regard to jurisdictional claims in published maps and institutional affiliations.

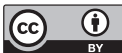

This work is licensed under a Creative Commons Attribution 4.0 International License. The images or other third party material in this article are included in the article's Creative Commons license, unless indicated otherwise in the credit line; if the material is not included under the Creative Commons license, users will need to obtain permission from the license holder to reproduce the material. To view a copy of this license, visit http://creativecommons.org/licenses/by/4.0/

(C) The Author(s) 2017 